\title{
Avaliação das boas práticas em Unidades de Alimentação Escolar da zona rural do município de Pelotas, RS
}

\author{
Evaluation of good practices in School Feeding Units \\ in the rural area of Pelotas, RS
}

\author{
Camila Irigonhé Ramos ${ }^{1}$, Marina Soares Valença ${ }^{2}$, \\ Nathalia Brandão Peter ${ }^{2}$, Ludmila Correa Muniz ${ }^{3}$
}

\section{Resumo}

\begin{abstract}
Objetivo: avaliar as boas práticas de alimentação, através de checklist, em Unidades de Alimentação e Nutrição (UAN) de escolas da rede municipal da zona rural da cidade de Pelotas, Rio Grande do Sul. Materiais e Métodos: trata-se de um estudo de corte transversal, de base escolar, do tipo censo, realizado nos anos de 2015/2016, com os gestores das escolas. Utilizou-se um checklist adaptado da Portaria Estadual 78 de 30 de janeiro de 2009, que foi constituído por 87 itens de avaliação, categorizados em 11 grupos. Para a avaliação dos itens críticos, seguiu-se os critérios de pontuação da RDC 275/2002. Cada UAN foi classificada como: Grupo 1, Grupo 2 e Grupo 3; cada grupo atende de 76 a $100 \%, 51$ a $75 \%$, até $50 \%$ dos itens, respectivamente. Os dados coletados foram duplamente digitados no programa EpiData 3.1 e posteriormente foram descritas as frequências absolutas e relativas no software Stata 12.1. Resultados: participaram da pesquisa 20 escolas, destas, com relação aos pontos críticos, $10 \%(\mathrm{n}=2)$ foram classificadas no Grupo $1 ; 30 \%(\mathrm{n}=6)$ no Grupo $2 \mathrm{e}$ $60 \%(\mathrm{n}=12)$ no Grupo 3. Quanto ao número total de itens, apenas $5 \%(\mathrm{n}=1)$ foi classificada no Grupo 1; $85 \%(n=17)$ no Grupo 2 e $10 \%(n=2)$ no Grupo 3. Conclusão: de maneira geral, a alimentação oferecida aos escolares, no que tange à sua produção, não atende as boas práticas de fabricação, gerando risco de contaminação, e não assegurando, desta forma, um dos princípios do Programa Nacional de Alimentação Escolar.
\end{abstract}

Palavras-chave: Boas práticas de fabricação. Lista de verificação. Controle de qualidade. Alimentação escolar.

1 Doutoranda em Ciências no Programa de Pós-graduação em Enfermagem da Universidade Federal de Pelotas, Pelotas, Rio Grande do Sul, Brasil. E-mail: mila85@gmail.com

2 Mestrado em Nutrição e Alimentos pelo Programa de Pós-graduação em Nutrição e Alimentos da Universidade Federal de Pelotas, Pelotas, Rio Grande do Sul, Brasil.

3 Doutorado em Epidemiologia pela Universidade Federal de Pelotas, Pelotas, Rio Grande do Sul, Brasil. Professora Adjunta da Faculdade de Nutrição da Universidade Federal de Pelotas, Pelotas, Rio Grande do Sul, Brasil. 


\title{
Abstract
}

\begin{abstract}
Objective: to evaluate the feeding practices, through a checklist made in public schools' Food and Nutrition Units (UAN) in the rural area of Pelotas, Rio Grande do Sul. Materials and Methods: this is a cross-sectional school-based census-type of study conducted in 2015/2016 with school managers. We used a checklist adapted from State Ordinance 78 of January 30, 2009, which consisted of 87 assessment items, categorized into 11 groups. For the evaluation of critical items, the scoring criteria of RDC 275/2002 were used. We classified each UAN as Group 1, Group 2, and Group 3; respectively, each group serves from 76 to $100 \%, 51$ to $75 \%$, up to $50 \%$ of items. The data collected were double entered in the software EpiData 3.1, and later the absolute and relative frequencies were described in the Stata 12.1 software. Results: twenty schools participated in the survey. Of these, $10 \%(n=2)$ were classified in Group 1; 30\% $(n=6)$ in Group 2 and $60 \%(n=12)$ in Group 3. Regarding the total number of items, only 5\%(n=1) was classified in Group $1 ; 85 \%(n=17)$ in Group 2 and $10 \%(n=$ 2) in Group 3. Conclusion: in general, the food offered to the students, regarding its production, does not meet the good manufacturing practices, generating a risk of contamination, and thus not ensuring one of the principles of the National School Feeding Program.
\end{abstract}

Keywords: Good manufacturing practices. Checklist. Quality control. School feeding.

\section{Introdução}

As ações de Segurança Alimentar e Nutricional (SAN) promovem o acesso a alimentos de qualidade e em quantidade adequada às necessidades nutricionais, sociais e culturais de toda a sociedade. Trata-se de um conceito abrangente, interdisciplinar e que objetiva a realização de práticas alimentares direcionadas à promoção da saúde e desenvolvimento da cidadania. $^{(1)}$ A SAN está intimamente ligada com o Direito Humano à Alimentação Adequada (DHAA) que é alcançado quando todas as pessoas têm acesso garantido e de maneira permanente à alimentação adequada e saudável.(2)

No Brasil, os estudantes da rede pública de educação básica possuem direito à alimentação escolar por meio do Programa Nacional de Alimentação Escolar (PNAE), um dos mais antigos na área de Alimentação, Nutrição e SAN. (3) $\mathrm{O}$ PNAE tem como objetivo principal garantir aos escolares o acesso ao alimento e práticas alimentares saudáveis, visando a redução de deficiências e excessos nutricionais, contribuindo, dessa forma, para o adequado crescimento e desenvolvimento biopsicossocial dos estudantes..$^{(4)}$ O programa, ainda, prevê ações de educação alimentar e nutricional, além da oferta de refeições saudáveis e seguras, que atendam as necessidades nutricionais dos alunos no ambiente escolar. ${ }^{(5)}$

A legislação brasileira orienta as ações e práticas do PNAE e determina os cuidados com a matéria-prima, o ambiente, os trabalhadores, a manipulação e as técnicas empregadas aos alimentos, que devem ser tomados com a alimentação oferecida pela escola. ${ }^{(6)}$ É importante atentar para a produção dessa alimentação, tendo em vista o controle de qualidade necessário para evitar contaminações e comprometimentos à saúde dos beneficiados. ${ }^{(7)}$

Vale ressaltar que, em locais onde há manipulação e produção de alimentos, existe maior risco de contaminação, direta ou indireta, relacionado aos manipuladores e locais de manipulação, e diante disso, existem legislações como a RDC 275 (2202) e RDC 216 (2004) que aprovaram os Procedimentos Operacionais Padronizados (POP) e as boas práticas para serviços de alimentação, a fim de nortear os gestores e os manipuladores de Unidades de Alimentação e Nutrição (UAN) a trabalhar mantendo a segurança em todas as etapas que envolvem a produção de alimentos. ${ }^{(8-9)}$ Logo, é importante lembrar que as Boas Práticas de Fabricação (BPF) constituem um processo permanente e dinâmico, que busca ao longo do tempo a qualidade dos produtos que 
estão sendo produzidos. ${ }^{(9)}$

Diante do exposto, o presente estudo teve por objetivo avaliar as boas práticas de alimentação, através de checklist, em UAN de escolas da rede municipal da zona rural da cidade de Pelotas, Rio Grande do Sul (RS).

\section{Materiais e Métodos}

Este estudo tem delineamento transversal, de base escolar, do tipo censo, realizado nos anos de 2015-16, com os gestores das 21 escolas municipais de ensino fundamental da zona rural de Pelotas, RS. Neste período, o município possuía cerca de 323 mil habitantes, sendo aproximadamente $7 \%$ residentes na zona rural. ${ }^{(10)}$ O presente estudo faz parte de uma pesquisa mais abrangente, que avaliou questões sobre a saúde e nutrição da comunidade escolar rural (escolares do ensino fundamental, pais/responsáveis, professores, trabalhadores da alimentação escolar e gestores) do município de Pelotas, RS.

Para avaliar as Boas Práticas de Fabricação (BPF) nas UAN das escolas, as informações foram coletadas através da aplicação de um questionário, contendo um checklist adaptado da Portaria Estadual 78 de 30 de janeiro de 2009, que tem por objetivo estabelecer procedimentos de boas práticas para serviços de alimentação, a fim de garantir as condições higiênico-sanitárias do alimento preparado. ${ }^{(11)} \mathrm{O}$ preenchimento do checklist ocorreu na própria UAN, nos horários de menor demanda, junto ao gestor responsável pela escola, por meio de observação direta do funcionamento, instalações físicas e análise documental.

O checklist foi adaptado da lista de verificação proposta no Anexo II da Resolução RDC 275/2002, e constituído por 87 itens de avaliação, categorizados em 11 grupos: (1) edificação, instalações, equipamentos, móveis e utensílios; (2) higienização de instalações, equipamentos, móveis e utensílios; (3) controle de pragas; (4) abastecimento de água; (5) manejo dos resíduos; (6) manipuladores; (7) matéria-prima, ingredientes e embalagens; (8) preparação do alimento; (9) exposição ao consumo do alimento preparado; (10) documentação e registro; (11) responsabilidade. Os itens em conformidade com a lista de verificação foram registrados como sim, os itens que não atendiam aos questionamentos foram registrados como não, e os itens não pertinentes à realidade do local foram registrados como não se aplica.

A avaliação dos itens críticos seguiu os critérios de pontuação da RDC 275/2002. Cada UAN foi classificada como: Grupo 1 (de 76 a 100\% de atendimento dos itens), Grupo 2 (entre 51 e 75\% de atendimento aos itens) e Grupo 3 (até 50\% de atendimento dos itens). Os dados coletados foram duplamente digitados no programa EpiData 3.1 e posteriormente foram descritas as frequências absolutas e relativas no software Stata 12.1.

O estudo foi submetido e aprovado pelo Comitê de Ética em Pesquisa da Faculdade de Medicina da Universidade Federal de Pelotas, sob número de protocolo 950.128. Para a realização da pesquisa dentro do ambiente escolar houve autorização da Secretaria Municipal de Educação e Desporto do município e também assinatura do Termo de Consentimento Livre e Esclarecido pelos gestores de cada escola participante.

\section{Resultados}

Dentre as 21 escolas municipais de ensino fundamental da zona rural do município de Pelotas, RS, 20 participaram da pesquisa, sendo nove escolas com ensino do primeiro ao quinto ano e 11 escolas com ensino do primeiro ao nono ano.

A Figura 1 apresenta a prevalência de conformidade dos itens críticos e do resultado geral da lista de verificação. Em relação aos pontos críticos, foi observado que somente $10 \%(n=2)$ das escolas participantes foram classificadas no Grupo 1, considerado a melhor classificação, visto que atende de 76 a $100 \%$ dos itens do checklist. Já no Grupo 2, 30\% $(\mathrm{n}=6)$ das escolas foram qualificadas atendendo de 51 a $75 \%$ dos itens, enquanto que no Grupo 3 foi observado que a maioria das escolas foi classificada com percentual de $60 \%(n=12)$, 
atendendo a menor quantidade de itens, até $50 \%$, do cheklist. É importante destacar o fato que somente duas escolas atenderam de 76 a $100 \%$ dos itens do checklist; e ainda, mais da metade foi classificada no Grupo 3, considerado o pior grupo por atender no máximo $50 \%$ dos itens.
Quanto ao número total de itens, apenas 5\% $(\mathrm{n}=1)$ foi classificada no Grupo 1, 85\% $(\mathrm{n}=17)$ no Grupo 2 e $10 \%(n=2)$ no Grupo 3. Observa-se que a maioria das escolas foi classificada no Grupo 2, atendendo a mais da metade do total de itens, de 51 a $75 \%$.

Figura 1 - Prevalência da conformidade com os itens críticos e do resultado geral da lista de verificação nas escolas municipais rurais de Pelotas, RS. 2015-16 (N=20).

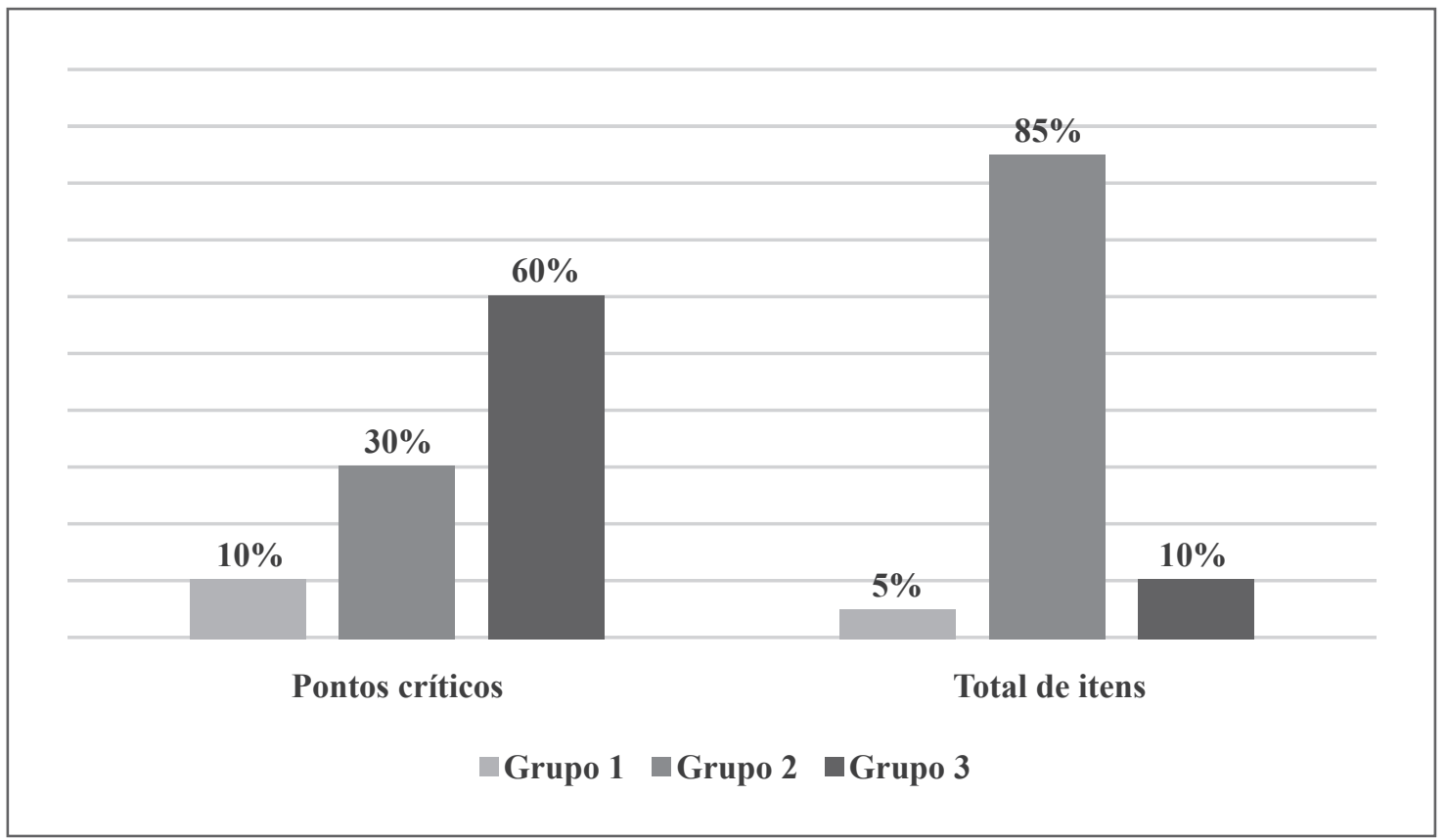

Fonte: Autoras

Analisando os 11 grupos dos itens que compõem a lista de verificação, é possível observar que poucas ou nenhuma escola foram classificadas no Grupo 1 e que somente o abastecimento de água não teve nenhuma escola classificada no Grupo 3. Destaca-se ainda que a maioria das escolas não possuem documentação e registro referentes ao manual de BPF e POP, que garantiria a qualidade e a conformidade dos alimentos com a legislação sanitária, proporcionando maior segurança alimentar a todos, bem como enquadram-se no Grupo 3 no que se refere ao item manejo de resíduos e responsabilidade (Tabela 1).

Em relação à situação das condições de edificação, instalação, equipamentos, móveis e utensílios a maioria das UAN não possuem teto com acabamento liso, impermeável e de fácil higienização. Também dispõem de equipamentos básicos, os quais atendem as necessidades demandadas. Porém, alguns equipamentos já estão em condições precárias havendo necessidade de substituição.

Nas portas existe proteção contra ratos e roedores e abertura telada em praticamente todas as escolas; porém, a vedação inferior de borracha não está sendo eficiente como barreira e em quatro escolas o controle de pragas não está sendo eficaz. Algumas portas não possuem molas, e o previsto pela legislação é ter largura e altura mínimas nunca inferiores a $1,00 \mathrm{~m}$ e $2,10 \mathrm{~m}$, respectivamente.

Os manipuladores de algumas escolas possuem uniforme composto por calça e camiseta, avental comprido e sapatos fechados e antiderrapantes, e touca de tecido que pode ser 
lavado e também existem toucas descartáveis. $\mathrm{Na}$ maioria dos casos mantém uma boa higiene pessoal, unhas curtas, sem esmaltes e não utilizam adornos.

No item preparação do alimento e exposição ao consumo do alimento preparado, observou-se que as cozinhas não possuem divisão física entre as áreas de trabalho. A preparação dos alimentos é distribuída entre as bancadas. Vale ressaltar que nem todas as escolas possuem bancadas de aço inoxidáveis. Estas não estão identificadas, visando minimizar o risco de contaminação cruzada durante o preparo com divisões que contemplem: área de pré-preparo de vegetais e frutas, pré-preparo de carnes e porcionamento, área de pré-preparo de cereais, sobremesas e expedição das preparações, área de cocção e área de higienização, área exclusiva para lavagem e desinfecção das mãos dos funcionários.

Tabela 1 - Distribuição das escolas municipais avaliadas na zona rural de Pelotas, RS, quanto aos grupos de verificação que compõem a Portaria 78/09-RS. 2015-16 (N=20).

\begin{tabular}{lccc}
\hline & $\begin{array}{c}\text { Grupo 1 } \\
(76-100 \%)\end{array}$ & $\begin{array}{c}\text { Grupo 2 } \\
(51-75 \%)\end{array}$ & $\begin{array}{c}\text { Grupo 3 } \\
(<50 \%)\end{array}$ \\
\hline Edificação, instalações, equipamentos, móveis e utensílios & 1 & 5 & 14 \\
Higienização de instalações, equipamentos, móveis e utensílios & 6 & 8 & 6 \\
Controle de pragas & 8 & 8 & 4 \\
Abastecimento de água & 0 & 20 & 0 \\
Manejo de resíduos & 0 & 5 & 15 \\
Manipuladores & 1 & 17 & 2 \\
Matérias-primas & 7 & 0 & 13 \\
Preparação do alimento & 0 & 6 & 14 \\
Exposição ao consumo do alimento preparado & 0 & 8 & 12 \\
Documentação e registro & 0 & 3 & 17 \\
Responsabilidade & 3 & 2 & 15 \\
\hline
\end{tabular}

Fonte: Autoras

\section{Discussão}

Por meio deste estudo foi possível verificar que as escolas participantes apresentaram dificuldades em atender mais de $50 \%$ dos itens considerados fundamentais para garantir as BPF. E, ainda, foi observado que a maioria das escolas, além de serem classificadas no Grupo 3 , que corresponde até $50 \%$ de atendimento dos itens, também apresentaram inadequações na edificação, instalações, equipamentos, móveis e utensílios; manejo de resíduos; matérias-primas; preparação do alimento; exposição ao consumo do alimento preparado; documentação e registro; e responsabilidade. $\mathrm{Na}$ perspectiva dos direitos humanos, o processo de como é feito o alimento é tão importante quanto o resultado do alimento preparado. E tais inadequações ferem o previsto no conceito de SAN, no que diz respeito à oferta de um alimento de qualidade; neste caso, mais especificamente a segurança microbiológica e transmissão de doenças. ${ }^{(13)}$

Corroborando os resultados deste estudo, outra pesquisa ${ }^{(14)}$ avaliou as condições higiênicas de escolas de ensino infantil e fundamental, por meio da verificação das boas práticas para as UAN escolares, quando as escolas de ensino fundamental atenderam uma média de $40,19 \%$ e $40,92 \%$ para as listas completas e reduzidas, respectivamente. A baixa adequação reflete a necessidade de desenvolver atividades para prevenir ações que possam comprometer a qualidade higiênico- 
sanitária da alimentação oferecida aos escolares. ${ }^{(14)}$

A literatura tem demonstrado que o não cumprimento das normas relacionadas com as BPF de alimentos é frequente, ressaltando a importância do profissional nutricionista para acompanhar e elaborar ações que possibilitem o controle e monitoramento das não conformidades encontradas. ${ }^{(14)}$ Inadequações de tipo estrutural foram verificadas em um estudo com escolas municipais de uma cidade do Recôncavo Baiano, onde foram observados, por exemplo, a falta de acabamento liso no teto e telas nas janelas contra insetos; armazenamento de alimentos junto com material de higiene e ausência de Manual de Boas Práticas. Demais pesquisadores ${ }^{(15)}$ também encontraram inadequações, principalmente na estrutura física, eressaltam que tais inconformidades podem comprometer a alimentação oferecida nas escolas estudadas.

Outros estudos ${ }^{(16-17)}$ ressaltam a inadequação dos manipuladores ao avaliar condições higiênicosanitárias do preparo dos alimentos em escolas. Encontraram que, apesar de grande parte das escolas estudadas apresentarem condições higiênico-sanitárias satisfatórias, ainda existe um alto índice de não adequação referente aos manipuladores, os quais não têm conhecimento sobre a necessidade da adequada higiene para elaboração da alimentação. Pesquisa realizada no município de Bayeux, na Paraíba, verificou que a maioria dos manipuladores não utilizava uniformes limpos, além de higienização precária do ambiente e equipamentos. Além disso, o controle de insetos e roedores não era realizado de maneira eficaz, bem como era incorreta a higienização de hortifrutigranjeiros. ${ }^{(17)}$ Uma pesquisa que avaliou as condições higiênico-sanitárias de cozinhas de creches públicas e filantrópicas na cidade de São Paulo, também encontrou inadequações que ofereciam riscos de contaminação à alimentação, sendo o mais importante dentre eles as ações dos manipuladores de alimentos, indicando a importância de treinamento para as pessoas que manipulam a alimentação dos escolares. ${ }^{(18)}$

Para atender aos preceitos que regem a SAN e o DHAA, o planejamento e execução do PNAE devem necessariamente ser mais rigorosos no que tange o monitoramento das ações de BPF de alimentos, para que, desta maneira, os estudantes recebam um alimento realmente de qualidade, e dentro do que o programa preza. ${ }^{(19)}$ Tratando-se de um programa de expansiva abrangência, em todo território nacional, longínquo e por estar inserido na escola, um local propício para ações de educação alimentar e nutricional, o PNAE deve ser visualizado pelos gestores como uma política de SAN, e, desta forma, necessita que todos os aspectos do conceito estejam sendo contemplados, inclusive o da segurança garantida pelas BPF;(20) tais aspectos também foram ressaltados em outras pesquisas que encontram inconformidades que indicam a necessidade de ações corretivas para diminuir o risco de contaminação e doenças aos escolares atendidos pelo PNAE, assim como para aumentar a eficiência do programa. ${ }^{(21)}$

É importante ressaltar que o estudo encontrou algumas limitações, dentre elas, a falta de disponibilidade de tempo do gestor da escola para responder o questionário da pesquisa, sendo inclusive o principal motivo para a recusa de uma escola para participar da pesquisa. É preciso, também, levar em consideração o fato de que muitas perguntas não foram respondidas por falta de conhecimento do gestor da escola. Outro fator importante são as questões em que obtiveram a resposta não se aplica, justamente por não ser possível mensurar alguns itens do questionário pela falta do instrumento necessário.

\section{Conclusão}

Com isso, é possível entender que a realização das BPF de alimentos é de difícil realização nas escolas públicas em todo o Brasil. Assim como na presente pesquisa, os estudos realizados com o objetivo de verificar as condições higiênicosanitárias de preparo da alimentação escolar encontraram inadequações em todos os setores, desde a estrutura física, manipulação dos alimentos, manipuladores e controle de pragas e vetores. 
Pode-se concluir que as produções das refeições oferecidas pelo PNAE de forma geral não atendem as BPF na alimentação escolar, e desta forma, existe o risco de contaminação e transmissão de doenças transmitidas de alimentos. Desta maneira, o PNAE não cumpre, na sua totalidade, com o seu papel de oferta de uma alimentação saudável, adequada e segura do ponto de vista da SAN e do DHAA.

\section{Referências}

1 Kepple AW, Segall-Corrêa AM. Conceituando e medindo a segurança alimentar e nutricional. Ciênc. Saúde Coletiva. 2011;16(1):187-99.

2 Maluf RS. Segurança alimentar e nutricional. Petrópolis - RJ: Vozes; 2009.

3 Peixinho AML. A trajetória do Programa Nacional de Alimentação Escolar no período de 2003-2010: relato do gestor nacional. Ciênc. Saúde Coletiva. 2013;18(4):909-16.

4 Brasil. Ministério da Educação. Cartilha Nacional da Alimentação Escolar. Brasília: MEC; 2015.

5 Brasil. Lei $n^{\circ}$ 11.947, de 16 de junho de 2009. Dispõe sobre o atendimento da alimentação escolar e do Programa Dinheiro Direto na Escola aos alunos da educação básica; altera as Leis nos 10.880, de 9 de junho de 2004, 11.273, de 6 de fevereiro de 2006, 11.507, de 20 de julho de 2007; revoga dispositivos da Medida Provisória no 2.178-36, de 24 de agosto de 2001, e a Lei no 8.913, de 12 de julho de 1994; e dá outras providências. Brasília: Presidência da República; 2009.

6 Brasil. Fundo Nacional de Desenvolvimento da Educação. Programa Nacional de Formação Continuada a Distância nas Ações do FNDE. 2a ed. Brasília: MEC; 2008.

7 Brasil. Manual de apoio para atividades técnicas do nutricionista no âmbito do PNAE: Programa Nacional de Alimentação Escolar. Brasília: FNDE; 2018.

8 Ferreira FS, Moura MS, Silveira ACP. Implantação de boas práticas de fabricação (BPF) em um laticínio de Piumhi-MG. PUBVET. 2011;5(13).
9 Veronezi CT, Caveião C. A importância da implantação das boas práticas de fabricação de alimentos. Rev Saúde Desenvol. 2015;8(4).

10 Prefeitura Municipal de Pelotas. Dados gerais. [Internet]. [citado $2018 \mathrm{dez}$ 5]. Disponível em: http://www.pelotas.rs.gov.br/cidade/dadosgerais.php

11 Rio Grande do Sul. Secretaria da Saúde. Portaria n. 78 de 28 de janeiro de 2009. Aprova a lista de verficação em boas práticas para serviços de alimentação, aprova normas para cursos de capacitação em boas práticas para serviços de alimentação e dá outras providências. Diário Oficial [do] Estado do Rio Grande do Sul, Porto Alegre, 2009 jan. 30; p. 35.

12 Burity V, Franceschini T, Valente F, Recine E, Leão M, Carvalho MF. Direito humano à alimentação adequada no contexto da segurança alimentar e nutricional. Brasília, DF: ABRANDH; 2010.

13 Declaração de Roma a Segurança Alimentar Mundial e Plano de Acção da Cimeira Mundial da Alimentação. [Internet]. Roma: World Food Summit; 1996 Nov 13-17. [citado 2019 jan 23]. Disponível em: http://www.fao.org/ docrep/003/w3613p/w3613p00.htm

14 Silva LHM, Brasil, CCB, Silveira ACM, Silveira JT, Moura FA, Martini CS, et al. Avaliação das condições higiênicas de escolas de ensino infantil e fundamental por meio da aplicação de listas de verificação. Segur. Aliment. Nutr. 2016;23(1):837-48.

15 Ramos AE, Borges NRS, Sousa AF. Avaliação das condições higiênico-sanitárias das cozinhas de escolas públicas de um município do semiárido nordestino. Rev. Interd. Ciên. Saúde. 2015 ago-out;2(3):64-73.

16 Soares DSB, Henriques P, Ferreira DM, Dias PC, Pereira S, Barbosa RMS. Boas práticas em unidades de alimentação e nutrição escolares de um município do estado do Rio de Janeiro - Brasil. Ciênc. Saúde Colet. 2018;23(12):4077-83. 
17 Lopes ACC, Pinto HR, Costa DCIO, Mascaranhas RJ, Aquino JS. Avaliação das boas práticas em unidades de alimentação e nutrição de escolas públicas do município de Bayeux, PB, Brasil. Ciênc. Saúde Colet. 2015;20(7):2267-75.

18 Oliveira MN, Brasil ALD, Taddei José AAC. Avaliação das condições higiênico-sanitárias das cozinhas de creches públicas e filantrópicas. Ciênc. Saúde Colet.2008;13(3):1051-60.

19 Valente FLS, Beghin N. Realização do direito humano à alimentação adequada e a experiência brasileira: subsídios para a replicabilidade. Brasília: ABRANDH; 2006.

20 Siqueira RL, Cotta RMM, Ribeiro CL, Sperandio N, Priore SE. Análise da incorporação da perspectiva do Direito Humano à Alimentação Adequada no desenho institucional do Programa Nacional de Alimentação Escolar. Ciênc. Saúde Colet. 2014;19(1):301-10.

21 Cardoso RCV, Góes JÂW, Almeida RCC, Guimarães AG, Barreto DL, Silva AS, et al. Programa nacional de alimentação escolar: há segurança na produção de alimentos em escolas de Salvador (Bahia)? Rev. Nutr. 2010;23(5):801-11. 